\title{
La fin des relations internationales?
}

\author{
Jean-Pierre Ferrier ${ }^{1}$
}

\begin{abstract}
* Cómo citar este artículo: Ferrier, J. P. (2021). La fin des relations internationales ? Relaciones Internacionales, 30(60), 126. https://doi.org/10.24215/23142766e126
\end{abstract}

Rassurons tout de suite le lecteur : cet article ne prévoit nullement, et son auteur ne souhaite pas du tout la disparition de cette excellente revue. Mais il redoute que l'étude des Relations internationales, notamment dans les Universités, ne devienne de plus en plus compliquée, voire impossible.

Il y a une trentaine d'années, I'universitaire français que j'étais se réjouissait du succès de l'un de ses anciens étudiants, devenu son collègue et resté toujours son ami, de l'autre côté de l'Atlantique, et qui parvenait à créer une revue dans le domaine de recherches qu'ils partageaient. J'en connaissais les difficultés. Mais j'étais assez optimiste, puisque je connaissais aussi les qualités et la volonté de Norberto Consani. II a réussi.

Son mérite n'est pas mince, car les Relations internationales deviennent de plus en plus difficiles à distinguer, donc à comprendre, donc à expliquer. Quand le futur fondateur de la revue était, par force et par choix, mon étudiant à Paris, les Relations internationales étaient encore une discipline balbutiante, mineure ; certes, il avait existé de remarquables historiens de la diplomatie et de la guerre, comme Jean-Baptiste Duroselle ; tant de juristes internationalistes exceptionnels, dont certains pouvaient être reconnus dans nos couloirs. Dans les années 1960, à l'Université de Paris, il y avait bien un cours d'Institutions internationales, dont l'essentiel était constitué par l'étude du fonctionnement des Organisations internationales. Les circonstances et les résultats de leur activité étaient pratiquement ignorés. Quant aux États, on apprenait en Droit international qu'ils étaient égaux et souverains, mais que tout ne se pasait pas toujours bien entre eux. Et, de la Science politique, on n'étudiait que l'existence de deux blocs antagonistes et d'un reste, appelé abusivement Tiers Monde. Osons le révéler : I'enseignement de Relations internationales était, pour les universités françaises, l'occasion d'accueillir des professeurs étrangers, souvent en exil.

\footnotetext{
${ }^{1}$ Ancien professeur, Université de Paris, France.
}

Editor: Juan Alberto Rial, Instituto de Relaciones Internacionales Facultad de Ciencias Jurídicas y Sociales (Universidad Nacional de La Plata)
Entidad editora: Relaciones

Internacionales, es una publicación del Instituto de Relaciones Internacionales (Facultad de Ciencias Jurídicas y Sociales (Universidad Nacional de La Plata Argentina)

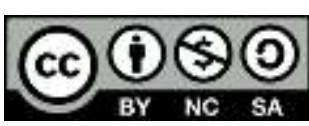

Reconocimiento-NoComercial Compartirlgual 4.0 Internacional (CC BY-NC-SA 4.0) 
Par exemple, mon prédécesseur, un remarquable internationaliste grec, avait un cours (passionnant) constitué en gros de deux parties : une "théorique " (les Relations internationales chez Thucydide et les auteurs grecs anciens, avec leur écho actuel) et une "pratique" (les conflits gréco-turcs)!

Était-il plus sage de s'attacher aux relations « réalistes » ? Et qu'entendre par là ? Essentiellement l'ensemble des relations, des éléments qui créaient des liens voulus ou non voulus, qui les confirmaient, les concrétisaient ou les contredisaient, entre différents acteurs : les États, les sujets non-étatiques du droit ou extérieurs au droit, et tous autres. Une approche vague, bien sûr, mais où les notions d'État ou de non-étatique délimitaient assez bien la réalité de la société internationale. Et des techniques diverses permettaient d'approcher mieux la réalité profonde, l'étude des idéologies, de la géopolitique, des relations économiques, etc.

Quand apparaît un nouveau domaine d'étude, il est bon que les approches soient diverses ; la conception de théories générales, par exemple, destinées à systématiser (malheureusement, à déformer parfois) les données observées, est très utile. Dans la revue "Relaciones Internacionales », je trouve un écho de toutes ces recherches. Surtout, l'évolution de la société, telle que les observateurs la perçoivent, y apparaissait et y apparaît encore très clairement, y compris dans ses obscurités, ses incertitudes. Or, celles-ci sont de plus en plus importantes. Officiellement, la base juridique de la matière, qu'il serait absurde d'ignorer, n'a pas beaucoup changé ; mais qui ose parler, sans restriction mentales ou exprimées, de "l'égalité » des États ? Au moins, restent-ils souverains ? Certains spécialistes ont noté, par exemple, que le terme de "souveraineté " est utilisé, dans le discours des dirigeants français, beaucoup plus souvent à propos de l'Union européenne, qui n'en aurait pas assez ou en userait mal, qu'à propos de la France elle-même...

Et comment distinguer clairement les États des éléments non-étatiques lors de la prise de décisions qui reviennent en principe aux premiers, alors que l'on sait très bien le rôle qu'ont pu jouer les sociétés multinationales, les groupes terroristes non-étatiques, les idéologies universalistes ou anti-universalistes, etc. ? La distinction entre la part de l'étatique et du non-étatique n'est pas toujours aisée : au sommet du pouvoir en Iran, avec des prolongements autour, l'ayatollah Khomeiny tenait-il son rôle, ses moyens d'action, sa place ... de son statut politique ou de son pouvoir religieux ? L'idéologie racialiste actuellement en vogue a imposé un critère absolu et unique à toutes les relations, passées et actuelles, donc aux relations internationales, celui de la culpabilisation et de la victimisation. Pour ses tenants (ses nouveaux ayatollahs), rien ne peut se comprendre ni s'expliquer sans cette relation totalement inégalitaire et nécessairement violente. La disparition de la lutte idéologique du $X X^{e}$ siècle comme facteur essentiel des relations internationales n'est donc, peut-être, que passagère : le contenu de l'idéologie a changé, non le rôle qu'elle peut jouer.

Les liens entre sociétés internationales et acteurs classiques de la vie internationale relèvent aussi d'études qui échappent largement à la discipline classique; ils sont encore plus secrets, ou au moins discrets, que les relations officielles et font surtout les délices de la presse et des prétendus connaisseurs de la "réalité cachée ». Le spécialiste des Relations internationales ne peut les ignorer; il ne peut que se fier à ce que d'autres peuvent 
apprendre, et essayer de faire la part du " politique » et du "commercial », sans aucune certitude. Est-ce donc, pour les spécialistes de cette discipline, le début de la fin ? Comme pour tout ce qui est lié à la science de la politique, la diversification des acteurs, des méthodes, des techniques et des règles juridiques exige au contraire des chercheurs à la fois conscients de leurs limites dans chaque domaine très spécialisé, et de leur utilité pour relier les différentes causes possibles aux événements constatés.

La revue "Relaciones Internacionales " reflète avec exactitude ce qui se passe dans notre monde, en cherche les causes, en évalue les conséquences possibles. Et c'est un grand mérite, car elle demeure claire dans sa grande diversité et vraiment internationale. Merci à elle et à son fondateur. 